Provided for non-commercial research and education use. Not for reproduction, distribution or commercial use.

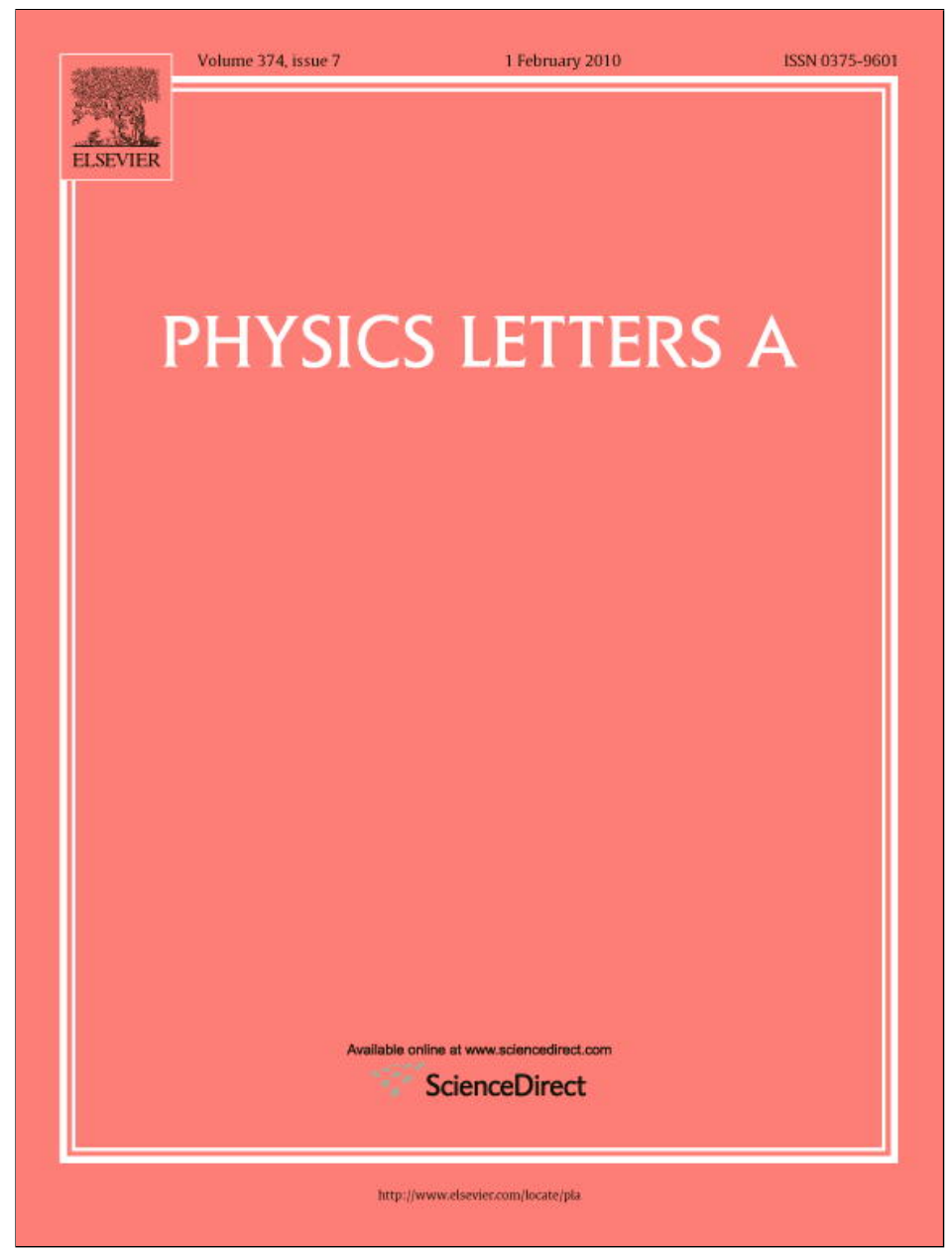

This article appeared in a journal published by Elsevier. The attached copy is furnished to the author for internal non-commercial research and education use, including for instruction at the authors institution and sharing with colleagues.

Other uses, including reproduction and distribution, or selling or licensing copies, or posting to personal, institutional or third party websites are prohibited.

In most cases authors are permitted to post their version of the article (e.g. in Word or Tex form) to their personal website or institutional repository. Authors requiring further information regarding Elsevier's archiving and manuscript policies are encouraged to visit:

http://www.elsevier.com/copyright 


\title{
Collisions and turbulence in optical rogue wave formation
}

\author{
G. Genty ${ }^{\mathrm{a}}$, C.M. de Sterke ${ }^{\mathrm{b}}$, O. Bang ${ }^{\mathrm{c}}$, F. Dias ${ }^{\mathrm{d}}$, N. Akhmediev ${ }^{\mathrm{e}}$, J.M. Dudley ${ }^{\mathrm{f}, *}$ \\ a Tampere University of Technology, Optics Laboratory, FI-33101 Tampere, Finland \\ ${ }^{\mathrm{b}}$ CUDOS ARC Centre of Excellence, School of Physics, University of Sydney, NSW 2006, Australia \\ c DTU Fotonik, Technical University of Denmark, DK-2800 Kongens Lyngby, Denmark \\ d Centre de Mathématique et de Leurs Applications (CMLA), ENS Cachan, France \\ e Optical Sciences Group, Research School of Physics and Engineering, Institute of Advanced Studies, The Australian National University, Canberra ACT 0200, Australia \\ ${ }^{\mathrm{f}}$ Département d'Optique P.M. Duffieux, Université de Franche-Comté, Institut FEMTO-ST CNRS UMR 6174, Besançon, France
}

\section{A R T I C L E I N F O}

\section{Article history:}

Received 10 November 2009

Accepted 4 December 2009

Available online 11 December 2009

Communicated by V.M. Agranovich

\begin{abstract}
A B S T R A C T
We discuss optical rogue wave generation in terms of collisions and turbulence processes. Simulations of picosecond pulse propagation in optical fibres show rogue soliton generation from either third-order dispersion or Raman scattering independently. Simulations of rogue soliton emergence with dispersive perturbation in the long-distance limit are also presented.
\end{abstract}

(c) 2009 Elsevier B.V. All rights reserved.

\section{Introduction}

There is currently much research interest into extreme-value fluctuations in optical fibre supercontinuum (SC) generation [1-4]. This has been motivated both by the possibility to obtain new fundamental insights into instabilities in fields such as hydrodynamics, Bose-Einstein condensates and optical filamentation [5-8], as well as by potential applications in generating white light spectra with controllable properties $[9,10]$. Although many aspects of fibre SC generation are now well-understood [11], these recent results have shown how noise-induced fluctuations can modify the dynamics so as to lead to very rare cases where high amplitude "optical rogue waves" are generated.

The initial description of optical rogue waves appeared in Ref. [1] and was applied to rare high amplitude soliton pulses generated on the long-wavelength edge of a broadband fibre SC spectrum. Amongst the mechanisms proposed to explain their appearance include the spontaneous emergence of a single high amplitude pulse from noise-induced modulation on a long pulse envelope and/or collisions between multiple solitons during subsequent propagation [2]. A distinguishing feature of these solitons is an extreme frequency-shifting trajectory towards longer wavelengths that separate them distinctly from the central region of the SC spectrum. This frequency separation allows their convenient experimental detection using spectral filtering and the measurement of highly skewed L-shaped probability distributions of soliton intensity [1] or energy [3]. Note that such skewed probability distributions with long tails can be considered to be a defining feature of rogue wave processes, since they predict the occurrence

\footnotetext{
* Corresponding author.

E-mail address: john.dudley@univ-fcomte.fr (J.M. Dudley).
}

of high amplitude events in the tails with greater probability than expected based on Gaussian statistics.

Other studies have identified a further class of fibre rogue wave phenomenon in the form of high-amplitude compressed pulses generated from modulation instability (MI). The temporal and spectral properties of these pulses can be described in terms of the rational Akhmediev Breather $(A B)$ solution of the nonlinear Schrödinger equation (NLSE) [12], and recent experiments have reported evidence for $A B$ dynamics during the initial evolution phase of quasi-continuous wave SC generation [13]. Because the AB subpulses are generated at an early stage of propagation before the emergence of distinct solitons, their spectral components do not appear distinctly at the edges of the SC spectrum, and their statistics must be analysed over the full SC bandwidth without filtering. But even without spectral filtering, collision and interaction effects between $A B$ pulses have been shown to lead to rogue wave characteristics and skewed probability distributions with extended tails [6].

Our objective in this Letter is to consider the propagation dynamics underlying the emergence of rogue wave characteristics for both the frequency-shifted solitons that are observed on the long wavelength edge of the fully developed SC spectrum, as well as the compressed $\mathrm{AB}$ pulses observed at the earlier propagation stage. For clarity in what follows, we use the terminology "rogue soliton" for the former case where high amplitude solitons appear distinctly isolated, and the more general term "optical rogue wave" for other cases. A particular goal of the Letter is to consider the effects of symmetry-breaking contributions to the NLSE dynamics introduced by stimulated Raman scattering (SRS) and third-order dispersion (TOD). Specifically, because it is well known that fibre solitons can undergo complex collision dynamics due to Ramanmediated interactions [14-16], it has been suggested that inelastic soliton collisions involving SRS are essential to the emergence 
(a) All terms
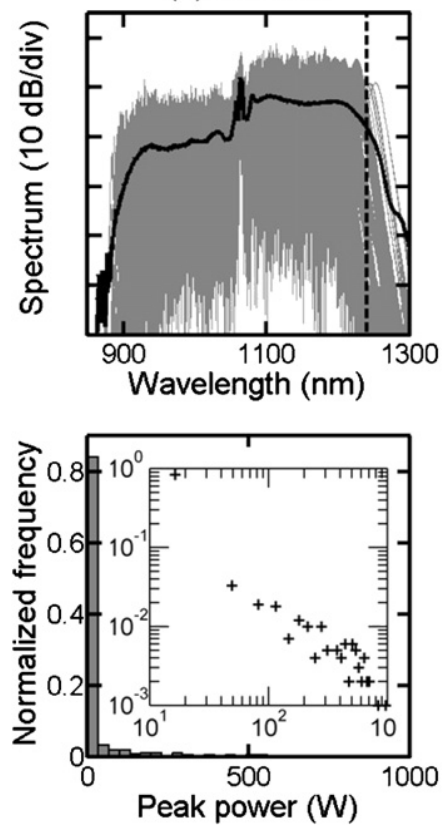

(b) TOD

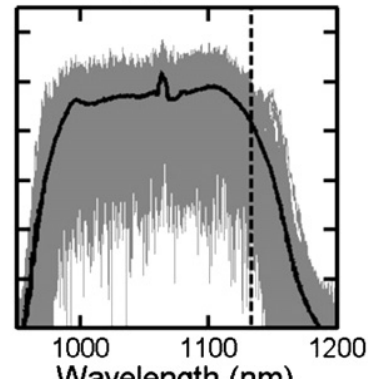

Wavelength $(\mathrm{nm})$

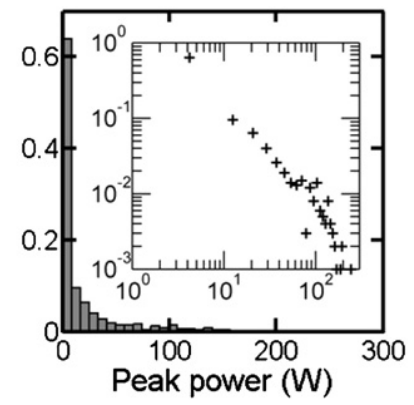

(c) SRS

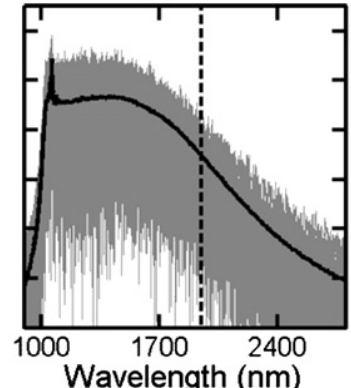

Wavelength $(\mathrm{nm})$

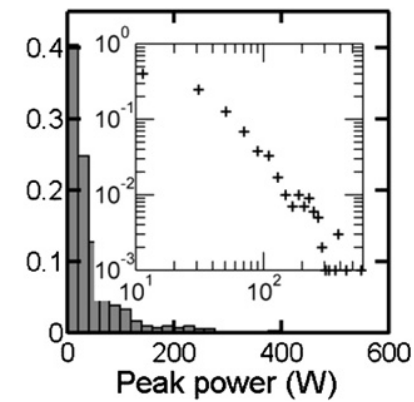

Fig. 1. Top graphs show individual realisations from an ensemble of 1000 simulations (grey curves) and calculated mean (black line) for simulations (a) based on full GNLSE with all perturbation terms; (b) simulations using NLSE with only TOD; (c) simulations using NLSE with only SRS. Bottom graphs show corresponding pulse height histogram using spectral filtering at the wavelengths shown as dashed lines in the upper figures. Insets: histograms in log-log scale.

of high-energy rogue solitons $[17,18]$. In this regard, however, although SRS and TOD both generate asymmetry such that coherent structures can acquire nonzero relative velocity and undergo collisions, we show here that a TOD perturbation only is sufficient to induce the emergence of rogue solitons and extreme-value statistics, and that Raman scattering is not in fact necessary. Indeed, we show that TOD on its own induces an intrinsic frequency downshift and dispersive wave trapping, effects also generally considered only in the context of intrapulse SRS [19]. Additional simulations using periodic boundary conditions show that these effects persist in the quasi-continuous-wave long-distance limit, and we discuss the resulting dynamics for this case in terms of soliton turbulence. In particular, we show how multiple collisions with sufficient propagation leads to the emergence of a single giant rogue soliton pulse from a low amplitude chaotic background.

\section{Numerical model}

Numerical simulations of nonlinear pulse propagation in fibres are typically based on a unidirectional generalized NLSE (or GNLSE) that can be written as [11]:

$$
\begin{aligned}
& \frac{\partial A}{\partial z}-\sum_{k \geqslant 2} \frac{i^{k+1}}{k !} \beta_{k} \frac{\partial^{k} A}{\partial t^{k}} \\
& \quad=i \gamma\left(1+i \tau_{\text {shock }} \frac{\partial}{\partial t}\right)\left(A(z, t) \int_{-\infty}^{+\infty} R\left(t^{\prime}\right)\left|A\left(z, t-t^{\prime}\right)\right|^{2} d t^{\prime}\right) .
\end{aligned}
$$

Here $A(z, t)$ represents the envelope of the electric field and the $\beta_{k}$ 's and $\gamma$ are the usual dispersion and nonlinear coefficients, respectively. The co-moving time $t$ is defined such that $t<0$ corresponds to a pulse leading edge, $t>0$ to a pulse trailing edge. The nonlinear response $R(t)=\left(1-f_{\mathrm{R}}\right) \delta(t)+f_{\mathrm{R}} h_{\mathrm{R}}(t)$ includes instantaneous and delayed SRS contributions. The fractional contribution of the Raman response is taken to be $f_{\mathrm{R}}=0.18$ and $h_{\mathrm{R}}$ is determined from the experimental fused-silica Raman cross-section [11]. The self-steepening or shock timescale $\tau_{\text {shock }}$ describes the dispersion of the nonlinearity. Of course, the basic NLSE model has $\tau_{\text {shock }}=0$ and $f_{\mathrm{R}}=0$ with only $\beta_{2}$ and $\gamma$ non-zero.

Rogue wave dynamics are typically observed when SC generation is induced from an initial stage of MI, and our simulations therefore consider picosecond pulse excitation under similar conditions to previous numerical studies and experiments [1-3]. In particular, we model $100 \mathrm{~W}, 5$ ps FWHM Gaussian pulses at $1060 \mathrm{~nm}$ propagating in $40 \mathrm{~m}$ of photonic crystal fiber with zero dispersion at $1040 \mathrm{~nm}$. These parameters are close to those used of the fiber used in the experiments reported in Refs. [1] and [4]. In Fig. 1(a), we first present simulation results modeling SC generation and rogue wave behavior using the full generalized NLSE model in Eq. (1). The dispersion coefficients at $1060 \mathrm{~nm}$ are: $\beta_{2}=-4.10 \times 10^{-1} \mathrm{ps}^{2} \mathrm{~km}^{-1}, \beta_{3}=6.87 \times 10^{-2} \mathrm{ps}^{3} \mathrm{~km}^{-1}, \beta_{4}=$ $-9.29 \times 10^{-3} \mathrm{ps}^{4} \mathrm{~km}^{-1}, \beta_{5}=2.45 \times 10^{-7} \mathrm{ps}^{5} \mathrm{~km}^{-1}, \beta_{6}=-9.79 \times$ $10^{-10} \mathrm{ps}^{6} \mathrm{~km}^{-1}, \quad \beta_{7}=3.95 \times 10^{-12} \mathrm{ps}^{7} \mathrm{~km}^{-1}, \quad \beta_{8}=-1.12 \times$ $10^{-14} \mathrm{ps}^{8} \mathrm{~km}^{-1}, \quad \beta_{9}=1.90 \times 10^{-17} \mathrm{ps}^{9} \mathrm{~km}^{-1}, \beta_{10}=-1.51 \times$ $10^{-20} \mathrm{ps}^{10} \mathrm{~km}^{-1}$. Other parameters were: $\gamma=0.011 \mathrm{~W}^{-1} \mathrm{~m}^{-1}$ and $\tau_{\text {shock }}=0.56$ fs. The input pulse soliton number for these parameters is $N \sim 147$. In our model, noise is included in the frequency domain through a one photon per mode spectral density on each spectral discretization bin [11], although we find from additional simulations that the precise form for the noise seeding is unimportant to the conclusions drawn below.

\section{Simulation results for picosecond pulse excitation}

Fig. 1 presents results for three different cases where we generated simulation ensembles of 1000 realizations using different noise seeds. In particular, we modeled propagation for: (a) the full GNLSE with all higher-order linear and nonlinear perturbations, (b) the NLSE model with only TOD perturbation (i.e. $f_{R}=0$, $\tau_{\text {shock }}=0, \beta_{k}=0, k>3$ ); (c) the NLSE model with only SRS perturbation (i.e. $f_{\mathrm{R}}=0.18, \tau_{\text {shock }}=0, \beta_{k}=0, k \geqslant 3$ ). The top graphs in the figure superpose the individual spectra from the ensemble (grey curves) together with the calculated mean (black line). The bottom graphs show the histogram of pulse amplitudes calculated from the corresponding time-series obtained by spectral filtering 

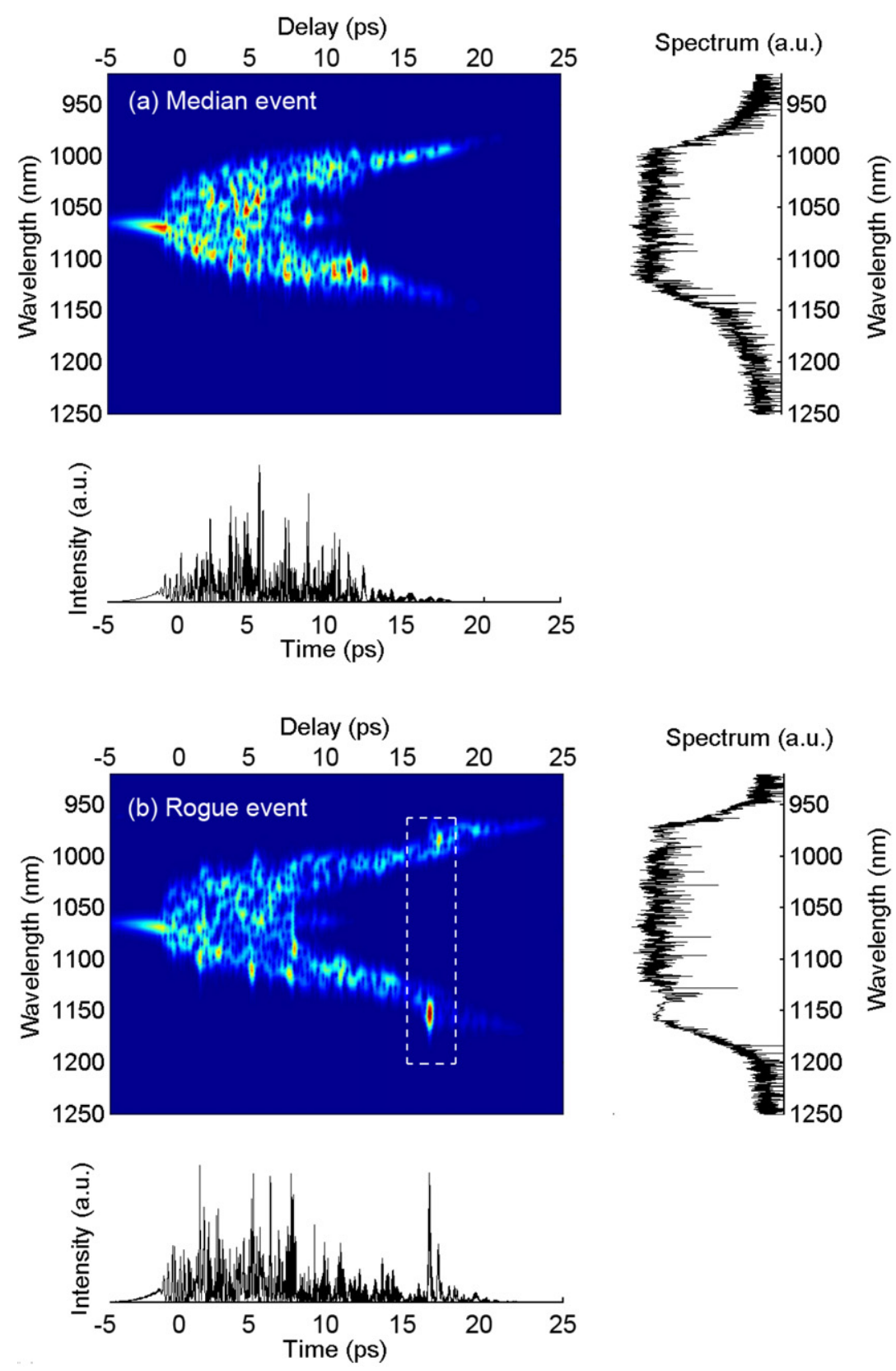

Fig. 2. Spectrogram representation of the output field for (a) a "median" and (b) a "rogue" event, extracted from simulations of Fig. 1(b) for NLSE simulations with only TOD perturbation. The spectrograms were calculated from the output field assuming a 100 fs gate function.

on the long wavelength edge at the wavelengths indicated (dashed lines). It is this filtering procedure that allows rogue soliton events to be isolated and identified [1-4].

The results in Figs. 1(a) and (b) use a spectral filter to select wavelengths above a point corresponding to $-10 \mathrm{~dB}$ intensity difference relative to the residual pump. Fig. 1(a) clearly shows the L-shaped heavy-tailed distribution characteristic of rogue soliton statistics, but Fig. 1(b) shows similar results with only a TOD perturbation, confirming that SRS is not a necessary prerequisite to observe this behavior. In the case of only an SRS perturbation, similar L-shaped statistics are observed with a similar spectral filter after longer propagation distances of $\sim 60 \mathrm{~m}$, or at the same propagation distance of $40 \mathrm{~m}$ but filtering further into the wings; these latter results are shown in Fig. 1(c). At this point we also remark that the spectral width in Fig. 1(c) is significantly wider than in Figs. 1(a) and (b) implying that the effects produced by SRS and
TOD are not simply additive. In fact, their simultaneous action depends strongly on the fibre parameters considered and the input pulse bandwidth [16]. For the particular case presented in Fig. 1(a) we may say that SRS is "suppressed" by the action of TOD.

The major conclusion to draw from Fig. 1 is that the output pulse characteristics and the propagation dynamics in the case of a NLSE with only TOD perturbation are qualitatively similar to those observed with the full GNLSE. To illustrate this, Fig. 2 plots output spectrogram results for two realisations taken from the ensemble in Fig. 1(b) and, in both cases, the general form of the spectrogram is similar to that typically observed for long-pulse SC generation studied using GNLSE modelling [11]. We also use this figure to highlight the difference between the output spectrograms for (a) a "median" event and (b) a "rogue" event, and we can clearly see that Fig. 2(b) shows a distinct rogue soliton pulse (localised around $1160 \mathrm{~nm}$ ) that has separated from the central region of 
(a) All terms
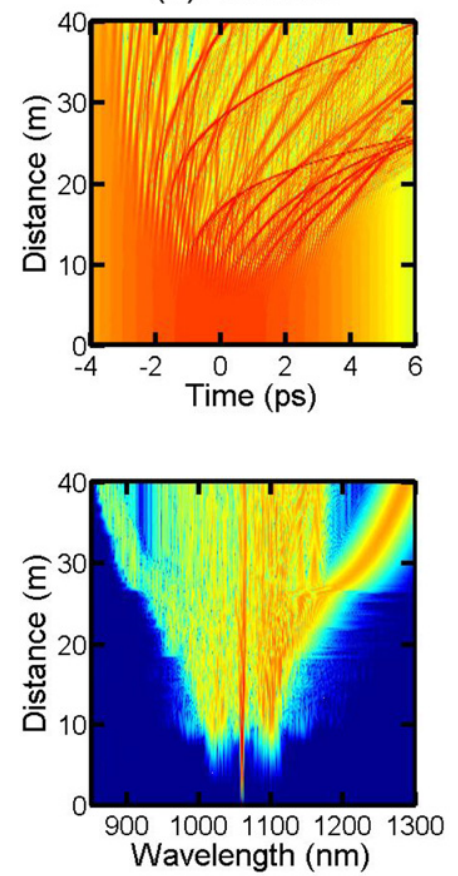

(b) TOD
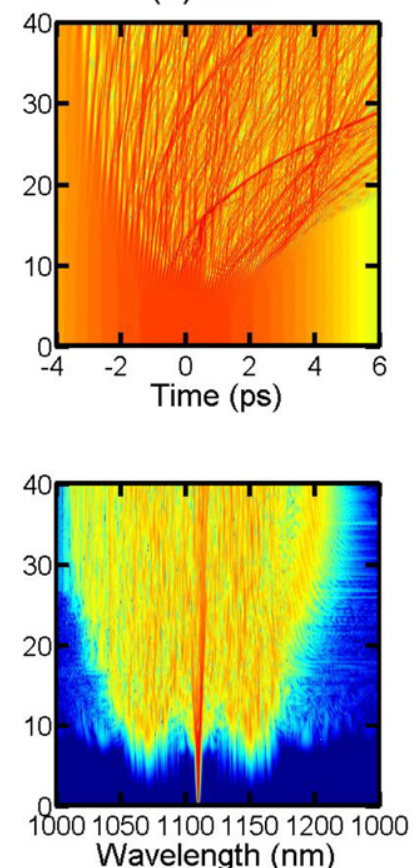

(c) SRS
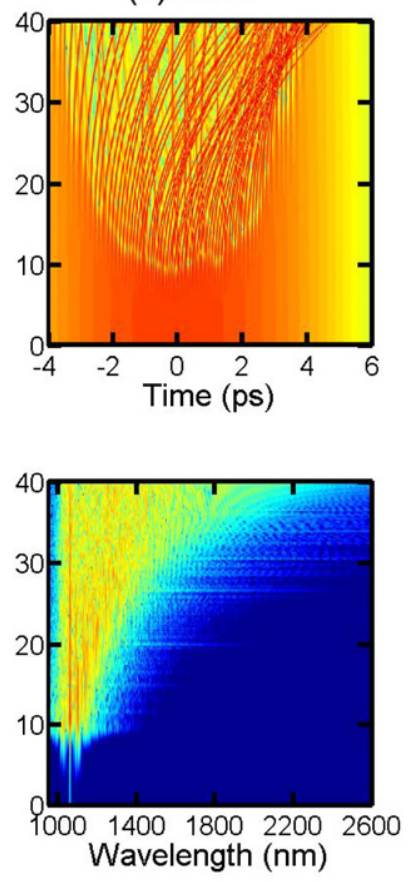

Fig. 3. Single-realisation results showing temporal intensity evolution using simulations (a) based on full GNLSE with all perturbation terms; (b) simulations using NLSE with only TOD; (c) simulations using NLSE with only SRS. The bottom figures show the corresponding spectral evolution. The trajectories in (a) and (b) were chosen to highlight rogue events. A detailed portion of the temporal trajectory in (b) is shown in Fig. 4(a).

the SC spectrum and appears on the trailing edge of the temporal profile. In this regard, we note that although temporal peaks at earlier times on the pulse envelope exhibit comparable amplitude, these arise from superposition across the full SC bandwidth and thus do not correspond to distinct rogue soliton trajectories. Importantly, we also note from this figure an associated trapped dispersive wave component [19] around $980 \mathrm{~nm}$ and we use a dashed box to highlight the coupled soliton-dispersive wave pair.

The observation of a rogue soliton and associated trapped dispersive wave with only a TOD perturbation to the NLSE is a significant new observation in the context of SC generation, suggesting that the long-wavelength frequency-shifting dynamics may in fact contain intrinsic contributions from non-Raman effects. These results obtained in the strongly nonlinear regime of SC regime complement earlier studies reporting TOD-induced frequency shifts in the context of near zero-dispersion wavelength soliton generation and dispersion-managed soliton systems [20,21].

An advantage of numerical simulations is the possibility to readily compare the influence of different contributing perturbations to particular dynamics. To this end, Fig. 3 presents single-shot realizations showing the evolution of the temporal intensity profile for: (a) the full GNLSE with all higher-order perturbations; (b) the NLSE model with only TOD perturbation; (c) the NLSE model with only SRS perturbation. The top figures show the temporal intensity evolution; the bottom figures show the spectral evolution. The realizations chosen correspond to cases where clear soliton trajectories are seen so that we can meaningfully compare the dynamics.

The qualitative evolution in Figs. $3(\mathrm{a})-(\mathrm{c})$ is similar. We see the emergence of distinct localized temporal solitons that follow different trajectories moving towards positive co-moving times in the figure (i.e. towards the pulse trailing edge, $t>0$ ). In the frequency domain, the spectral evolution of the GNLSE and TOD cases in Figs. 3(a) and (b) are very similar in showing clear spectral localization of the extreme soliton trajectories; an important remark here is that these solitons retain their coherent structure over the distances considered once they have separated temporally and spectrally relative to the residue of the injected pulse; our simulations do not show any evidence of spontaneous collapse as reported in Ref [1]. In Fig. 3(c) with only SRS, the generated solitons span over an increased wavelength range and their spectral separation is less distinct; but as shown above in Fig. 1(c), however, filtering can still reveal the presence of long-tailed statistics, as would be expected based on the qualitatively-similar time-domain evolution to Figs. 3(a) and (b).

The dynamics for the cases shown in Fig. 3 involves a series of distinct localized ultrashort pulses emerging from an initial pulse envelope after around $10 \mathrm{~m}$ propagation, followed by a complex series of interaction, collision and frequency shifting processes. The specific results in Figs. 3(b) and (c) for TOD and SRS acting separately allow us to draw further conclusions about the role of collisions for these two cases. Firstly, we note that the primary effect of TOD on solitons is a constant velocity shift they acquire which is proportional to their energy [22]. Thus, in the absence of any other process, solitons move along linear trajectories (with slope depending on energy) after being generated from the continuum. However, the effect of collisions is to modify the energy of the propagating solitons and induce a subsequent change in their velocities. With our choice of co-moving frame, solitons which gain more energy are delayed more (i.e. they move slower towards $t>0)$. As a soliton undergoes multiple collisions and gains energy, it progressively accumulates additional delay and decelerates, resulting in a temporal trajectory with concave-down curvature as is clearly visible in Fig. 3(b). In contrast, solitons in purely Raman media experience a constant deceleration proportional to the soliton amplitude [23]. In the absence of any other process, this yields parabolic trajectories towards increasing delays $(t>0)$. Thus, even if initially all solitons had identical velocities, this deceleration shifts them quickly to the right relative to the centre of the initial pulse. Although the number of collisions could be similar to the case of TOD only, the energy exchange rate in collisions is lower 
(a) NLSE with TOD and noise
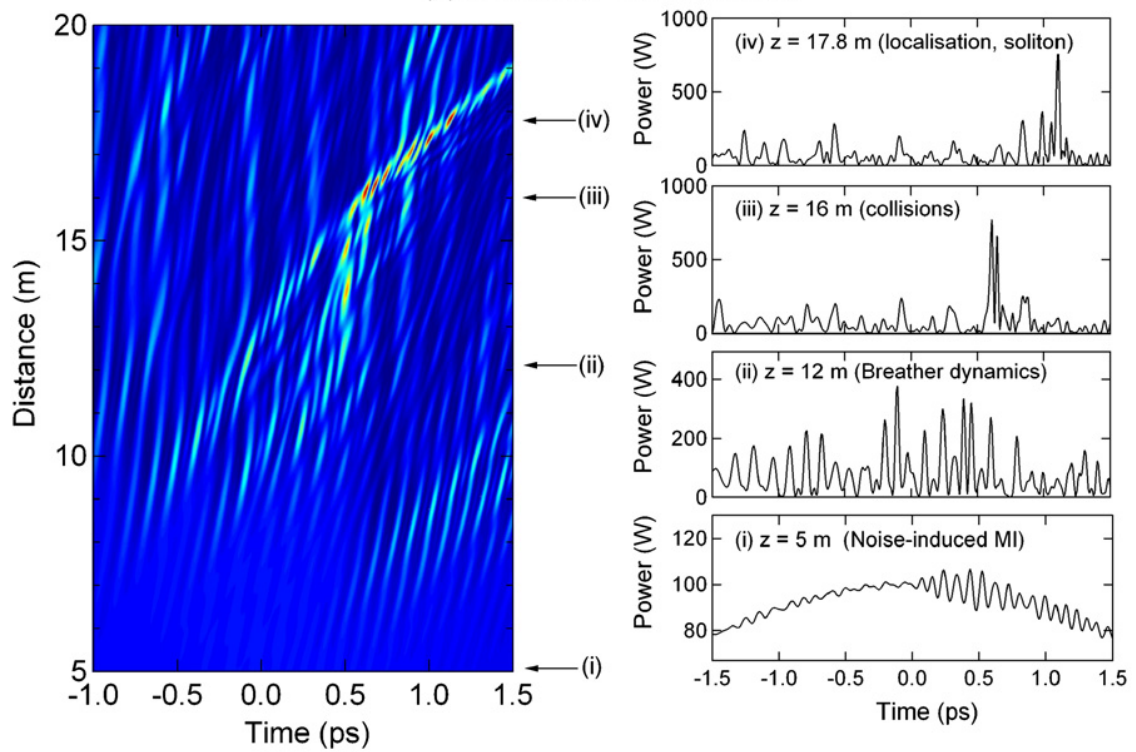

(b) NLSE with noise
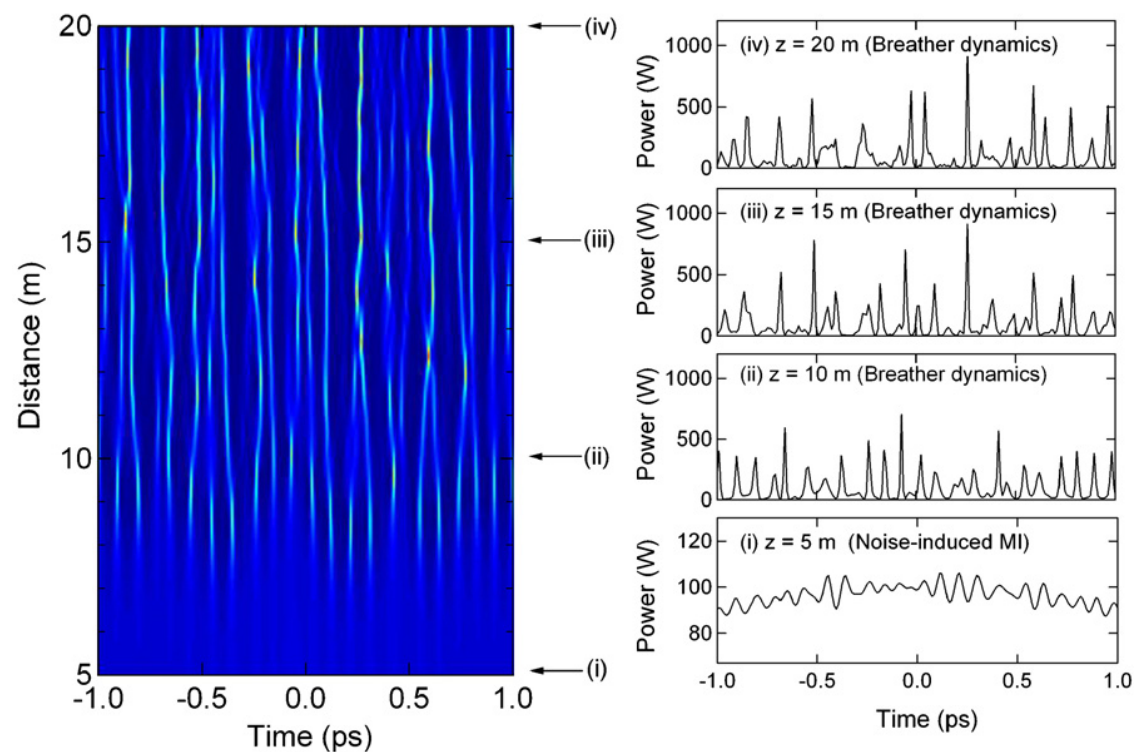

Fig. 4. (a) Detailed view of propagation in the NLSE with noise and TOD over the region indicated in Fig. 3(b) including line profiles of intensity at propagation distances as indicated. (b) shows the evolution for the case without the effect of TOD. Both figures show initial evolution associated with noise-induced MI and the appearance of high-contrast pulses. In (a) when TOD is included in the simulations, there is an additional phase of drift, interactions, collisions and localisation towards the appearance of solitons.

and the energy growth for the larger solitons is relatively small. This is clearly visible in Fig. 3(c). Thus, solitons do not accumulate energy due to collisions as much as in the TOD case. From these simple considerations, we can conclude that TOD may provide better conditions for generation of high energy solitons than Raman effect merely because of the higher rate of the energy exchange in collisions. Of course, we note that although these conclusions are likely to be general for silica fibres with typical dispersion curves as considered here, the propagation dynamics in fibers with different Raman responses and/or more complex dispersion curves may require additional consideration.

Concerning the question of what particular mechanisms cause particular rogue soliton trajectories to emerge from an input pulse undergoing MI, we note that this remains an open problem. However, in showing that only a TOD perturbation to the NLSE can lead to this behaviour, our results establish links with previous studies in other (discrete) NLSE systems where it has been nonintegrability and asymmetry rather than any one specific physical effect that has been identified as a factor underlying nonlinear localisation [22-26].

In the particular context of fiber SC generation, we have inspected a large number of additional simulations seeded by different noise conditions. For both the TOD-only and Raman perturbation cases in Figs. 3(a) and (b), we identify that the emergence of rogue soliton trajectories in the long-distance limit is associated with evolution trajectories where distinct sub-pulses formed from an initially modulated envelope undergo very apparent collision and/or interaction events at an early stage of the dynamical propagation. To examine this in more detail, Fig. 4(a) plots an expanded region of Fig. 3(b). We use a pseudocolor representation of the temporal intensity evolution, and plot the field intensity profiles at selected distances as shown. For comparison, Fig. 4(b) plots 
similar results but for the case of NLSE dynamics only without TOD or Raman.

We discuss the results in Fig. 4 in terms of several distinct regions where different physical effects come into play. Firstly, in subfigure (i) of Fig. 4(a) at $z=5 \mathrm{~m}$, we see how the input pulse noise induces a temporal modulation instability on the propagating pulse envelope. Although the MI is induced by broadband noise, the modulation contains a dominant frequency component at the peak of the MI gain $\Omega_{M I} / 2 \pi=\left(2 \gamma P_{0} /\left|\beta_{2}\right|\right)^{1 / 2} / 2 \pi=11.7 \mathrm{THz}$ corresponding to temporal period $\sim 86 \mathrm{fs}$. With subsequent propagation to around $12 \mathrm{~m}$, subfigure (ii) of Fig. 4(a) shows how this modulation develops into a train of high-contrast pulses, and it is this propagation regime that can be interpreted physically in terms of the initial growth phase of Akhmediev breather evolution [13]. In fact, ideal Akhmediev breather structures correspond to analytic solutions of the NLSE for MI induced by a narrowband field, but even for the case of spontaneous MI developing from broadband noise, a temporally-modulated pulse envelope nonetheless exhibits characteristic breather properties such as high pulse contrast and the presence of neighboring low-amplitude sub-pulses. In fact, the equivalent soliton number of these breather structures is approximately unity and thus they naturally evolve towards fundamental solitons in the presence of perturbations. For our case, we can see how TOD (which lifts the group-velocity degeneracy of the different sub-pulses with different spectral content and temporal amplitudes) induces subsequent collisions and interactions during this evolution from the initial breather formation phase. The pseudocolor plot shows a particular collision event occurring at the point $(0.5 \mathrm{ps}, 16 \mathrm{~m})$ which yields significant localisation in the form of an emerging high-amplitude pulse as shown in subfigures (iii) and (iv) of Fig. 4(a). It is the subsequent evolution of these localised structures formed at this stage that can lead to the ultimate emergence of near ideal high amplitude solitons with propagation out to greater distances.

Another feature that is apparent from the pseudocolor plot is that the evolution in the presence of TOD is associated with the temporal drift of the MI sub-pulse structure from the pulse leading edge $(t<0)$ towards the pulse trailing edge $(t>0)$. The drift behavior can be seen very clearly by comparing Figs. 4(a) and (b). In fact, for the latter case when no TOD is present, although there is a similar evolution of initial MI towards high-contrast breather structures as in Fig. 4(a), the lack of asymmetry in the NLSE precludes drift-induced collisions with subsequent propagation.

Although there has been a recent proposal to interpret instabilities in rogue wave generation in terms of convective processes [27], we do not consider this in detail here. Nonetheless, we can see from our results that the slope of the temporal drift itself varies across the temporal profile and the particular collision that leads to the emerging localized soliton appears to arise from a collision between drifting sub-pulses from the leading edge $(t<0)$ and more slowly-moving sub-pulses on the trailing edge $(t>0)$. In addition to the central role of TOD, preliminary simulations suggest that both the chaotic initial conditions and the power variation across the input envelope due to the use of pulsed excitation also contribute to the quantitative value of the drift. In terms of subsequent evolution towards rogue events, the precise nature of the resulting probability distribution will depend in detail on whether the breather dynamics or later soliton dynamics are dominant at the particular fiber length considered. But for both cases, our results show that collisions are essential factors, as they permit energy exchange between the emerging pulses so that those of higher amplitude gain more energy at the expense of the lower. These numerical results extend previous theoretical studies of TOD-induced energy exchange for certain classes of soliton collisions [28], and we now discuss their particular extension to an interpretation in terms of soliton turbulence.

\section{Simulation results for periodic boundary conditions - soliton turbulence}

In an optical context, the results in Figs. 3 and 4 are consistent with earlier studies where rogue soliton emergence was linked to collisions [17], but they extend this previous work by showing that this can occur with only TOD perturbation. Additional studies show that the effects of TOD-induced self-frequency shift, dispersive wave trapping and the emergence of a rogue soliton from collision dynamics persist when the simulations are extended to a quasicontinuous-wave long-distance limit. In particular, we perform additional simulations with an NLSE with TOD model, but impose periodic boundary conditions which allow multiple collisions to occur over long propagation distances. Physically, multiple simulations of this type with different noise seeds would be expected to provide a realistic model of evolution for quasi-continuous-wave or continuous-wave excitation, and studies using an NLSE model with nonlinear perturbation by Zakharov have shown that it leads to a state of "soliton turbulence" [29].

The regime of soliton turbulence describes propagation where an initial phase of multiple soliton formation from a noisy background is followed by the emergence of a single large soliton that extracts energy from the background and acts as a statistical attractor [30-32]. In fact, this occurs as a result of the general principle that in collisions of solitons in non-integrable systems (where the number of solitons is not conserved) the stronger soliton becomes stronger while the weaker one loses energy [33]. This process can be viewed as a manifestation of the principle of "survival of the fittest" in nonlinear dynamics. Clearly, for such growth to be significant requires a multiplicity of collisions. Motivated by our results above for pulsed excitation that show how TOD can induce breather and soliton collisions, we now consider the dynamics of soliton turbulence with an ideal cubic nonlinearity and only TOD perturbation and show that this leads to an appreciable growth of a single soliton.

These simulations use the same fiber parameters as above, but consider input pulse duration of 10 ps (FWHM) and a propagation distance of $500 \mathrm{~m}$. Periodic boundary conditions were imposed over a time window of 20 ps. Fig. 5 shows the results obtained, plotting temporal intensity (top) and the corresponding spectrum (bottom) at selected distances of 20,70 , and $500 \mathrm{~m}$. From the temporal trace it can be seen how a large-amplitude localized structure emerges progressively from an ensemble of waves with significantly lower amplitude, and the emergence of this largeamplitude localized structure is correlated to the gradual broadening of the spectrum both on the long-wavelength and shortwavelength edges. The calculated field spectrograms at the same distances shown in Fig. 6 provide further insight, and clearly show the emergence of one distinct pulse in the time-frequency domain from the low amplitude background. In fact, we have found that the emerging large-amplitude pulse is well-fitted by a hyperbolicsecant profile and has soliton order near-unity, confirming the interpretation of a giant rogue soliton pulse emerging from and propagating amidst an otherwise low amplitude noise background.

Fig. 7 shows additional characteristics of this soliton pulse as it emerges from the background where, as a function of propagation distance, we isolate the soliton pulse and calculate its center wavelength and energy. Significantly, we stress again that even though Raman scattering is not included in the simulations, we still observe a steady frequency shift to longer wavelengths. The TOD-induced frequency shift was also noted above in the simulations in Fig. 1(b), but the results here with periodic boundary conditions and multiple collisions allow us to more clearly see how this dynamics is also associated with a corresponding increase in pulse energy. In this context, note that the pulse duration of the emerging soliton remains constant at around 150 fs (FWHM) even 

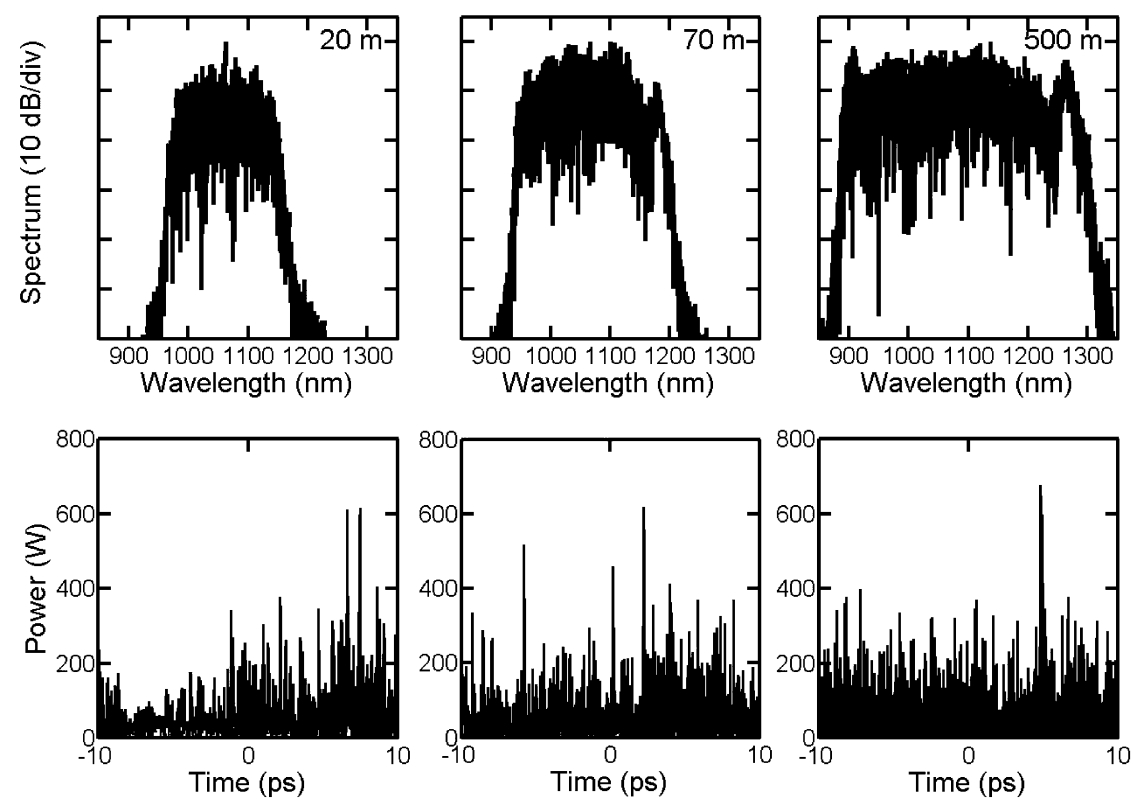

Fig. 5. Spectral (top) and temporal (bottom) profiles of evolving SC field at selected distances as shown in a long-distance limit with multiple collisions. Simulations use an NLSE with only TOD perturbation.
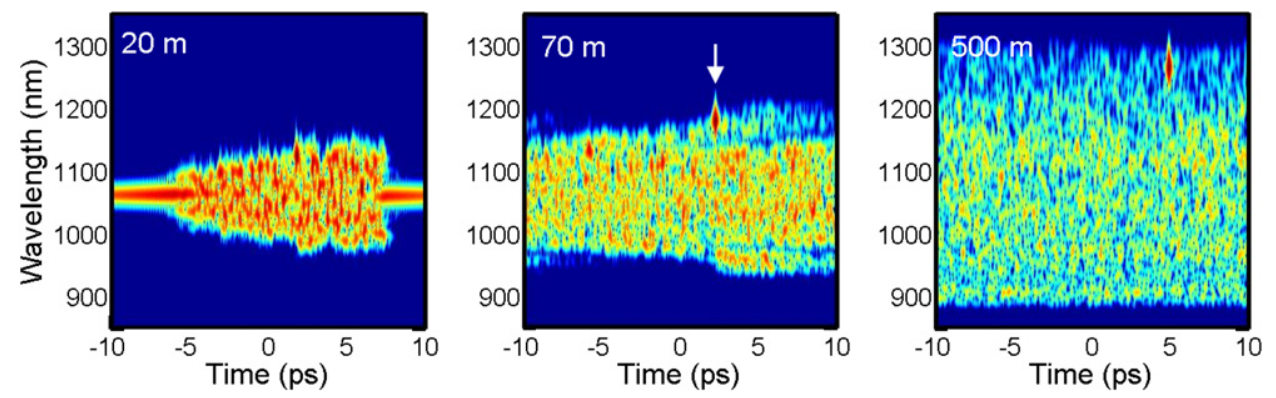

Fig. 6. Spectrogram evolution at distances shown corresponding to the results in Fig. 5 . The emergence of a single localised soliton structure from multiple collisions becomes apparent after around $70 \mathrm{~m}$ (indicated by the arrow). A logarithmic colormap is used to highlight the low amplitude structure.
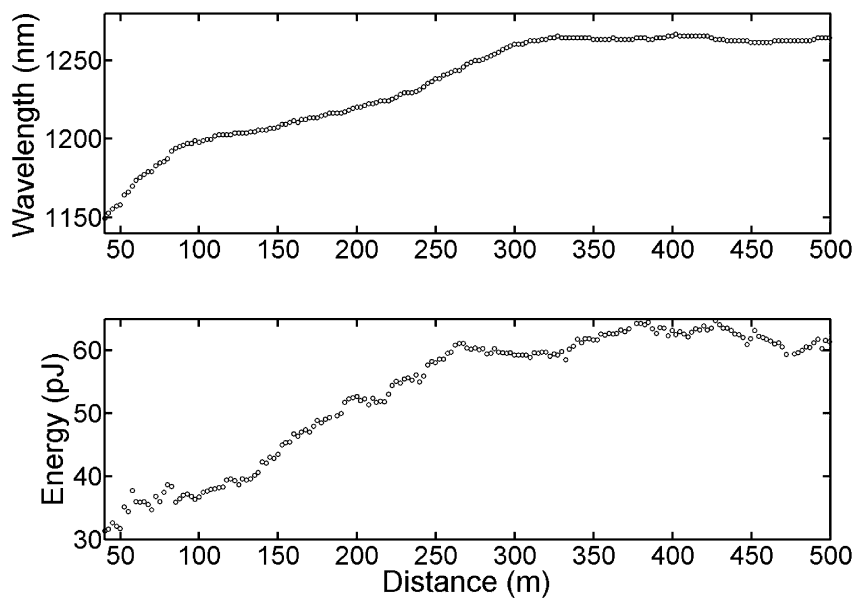

Fig. 7. Evolution of emergent soliton central wavelength and energy as a function of propagation distance for results in Figs. 5 and 6.

as its energy increases. The shift to longer wavelengths (larger $\left|\beta_{2}\right|$ ) can then be viewed as a consequence of the soliton area-theorem that requires that a constant energy-to- $\beta_{2}$ ratio be maintained. Also of interest is the apparent saturation in the wavelength shift and energy for propagation distances beyond $\sim 300 \mathrm{~m}$. Note that qualitatively similar self-regulating behaviour has been observed using statistical approaches to study self-organisation and asymptotic persistence of large-amplitude solitary-waves in other classes of non-integrable NLS systems [30].

\section{Conclusions}

There are several main conclusions to make based on the results above. Firstly, our results have shown that SC generation described by an NLSE model perturbed only by TOD can lead to large amplitude localized optical rogue soliton and rogue wave structures. Generally speaking, any single perturbation that destroys the integrability of the NLSE is sufficient to observe "survival of the fittest" soliton amplification as a result of collisions. In order to observe the significant growth of a particular soliton the dynamics has to provide multiplicity of collisions, and our results show that this can occur purely with the dispersive TOD perturbation without the need for higher order nonlinearity or Raman scattering.

Our simulations also yield the qualitative observation that collisions between emerging breather structures from an envelope undergoing MI appears to be a precursor to the formation of rogue soliton pulses, and it is possible to conjecture that a difference in relative velocities between breathers formed across an initial wave field is an important (perhaps necessary) condition for rogue soliton emergence. In fact, although the rogue solitons in fibre optics separate distinctly from the initial field be- 
cause of self-frequency shifting, it is likely that the emergent and colliding breather structures provide a closer analogy with nonlinearity-induced rogue waves on the ocean. This proposal may have wide impact, as it implies that the seeds of rogue wave formation in optics as well as hydrodynamics may be found by identifying particular wave field characteristics (e.g. initial conditions [34], noise frequency content $[9,17]$ ) or specific medium properties (e.g. topography) that favor significant breather velocity gradients.

Secondly, our simulations carried out in a long distance limit have shown that multiple interactions and collisions result in the formation of a turbulent background field upon which a giant soliton pulse emerges as a result of energy exchange between an emerging localized structures and the chaotic background. This result confirms earlier important suggestions of the universality of this mechanism [22-26]. Indeed, in addition to providing improved insight into the mechanisms of hydrodynamic rogue wave formation, similar localisation processes may play central roles in the formation of biomolecular structures [35]; the study of the nonlinear dynamics in such systems is a developing area of research [35-38].

\section{Acknowledgements}

We thank the Academy of Finland (Research grants \#121953, \#130099, and \#1332279), the Institut Universitaire de France, and the French Agence Nationale de la Recherche project MANUREVA (ANR-08-SYSC-019) for support. The work of N.A. is supported by the Australian Research Council (Discovery Project No. DP0663216).

\section{References}

[1] D.R. Solli, et al., Nature 450 (2007) 1054.

[2] J.M. Dudley, G. Genty, B.J. Eggleton, Opt. Express 16 (2008) 3644.
[3] C. Lafargue, J. Bolger, G. Genty, F. Dias, J.M. Dudley, B.J. Eggleton, Electron. Lett. 4 (2009) 217.

[4] M. Erkintalo, G. Genty, J.M. Dudley, Opt. Lett. 34 (2009) 2468.

[5] N. Akhmediev, A. Ankiewicz, M. Taki, Phys. Lett. A 373 (2009) 675.

[6] N. Akhmediev, J.M. Soto-Crespo, A. Ankiewicz, Phys. Lett. A 373 (2009) 2137.

[7] Yu.V. Bludov, V.V. Konotop, N. Akhmediev, Phys. Rev. A 80 (2009) 033610.

[8] J. Kasparian, P. Béjot, J.P. Wolf, J.M. Dudley, Opt. Express 17 (2009) 12070.

[9] D.R. Solli, C. Ropers, B. Jalali, Phys. Rev. Lett. 101 (2008) 233902.

[10] G. Genty, J.M. Dudley, IEEE J. Quantum Electron. 45 (2009) 1331.

[11] J.M. Dudley, G. Genty, S. Coen, Rev. Mod. Phys. 78 (2006) 1135.

[12] N. Akhmediev, V.I. Korneev, Theor. Math. Phys. 69 (1986) 1089.

[13] J.M. Dudley, G. Genty, F. Dias, B. Kibler, N. Akhmediev, Opt. Express 17 (2009) 21497.

[14] B.A. Malomed, Phys. Rev. A 44 (1991) 1412

[15] F. Luan, D.V. Skryabin, A.V. Yulin, J.C. Knight, Opt. Express 14 (2006) 9844

[16] M.H. Frosz, O. Bang, A. Bjarklev, Opt. Express 14 (2006) 9391.

[17] G. Genty, J.M. Dudley, B.J. Eggleton, Appl. Phys. B 94 (2009) 187; See also arXiv:0809.2388v1 [physics.optics], 2008.

[18] A. Mussot, A. Kudlinski, M.I. Kolobov, E. Louvergneaux, M. Douay, Majid Taki, Opt. Express 17 (2009) 17010.

[19] A.V. Gorbach, D.V. Skryabin, Nature Photon. 1 (2007) 653.

[20] M. Desaix, D. Anderson, M. Lisak, Opt. Lett. 15 (1990) 18.

[21] T.I. Lakoba, G.P. Agrawal, J. Opt. Soc. Am. B 16 (1999) 1332

[22] N. Akhmediev, M. Karlsson, Phys. Rev. A 51 (1995) 2602.

[23] N. Akhmediev, W. Krolikowski, A.J. Lowery, Opt. Commun. 131 (1996) 260

[24] T. Dauxois, M. Peyrard, Phys. Rev. Lett. 70 (1993) 3935.

[25] O. Bang, M. Peyrard, Phys. Rev. E 53 (1996) 4143.

[26] O. Bang, P.D. Miller, Opt. Lett. 21 (1996) 1105

[27] A. Mussot, A. Kudlinski, M.I. Kolobov, E. Louvergneaux, M. Douay, Majid Taki, Mechanisms of rogue wave formation, arXiv:0903.4522, 2009

[28] B.A. Malomed, Phys. Rev. A 43 (1991) 3114.

[29] V.E. Zakharov, N. Pushkarev, V.F. Shvets, V.V. Yan'kov, JETP Lett. 48 (1988) 83.

[30] R. Jordan, C. Josserand, Phys. Rev. E 61 (2000) 1527.

[31] B. Rumpf, A.C. Newell, Phys. Rev. Lett. 87 (2001) 054102.

[32] V. Zakharov, F. Dias, A. Pushkarev, Phys. Rep. 398 (2004) 1.

[33] A.V. Buryak, N. Akhmediev, Phys. Rev. E 50 (1994) 3126.

[34] N. Akhmediev, J.M. Soto-Crespo, A. Ankiewicz, Phys. Rev. A 80 (2009) 043818.

[35] M. Guéron, M. Kochoyan, J.L. Leroy, Nature 328 (1987) 89.

[36] M. Peyrard, S.C. Lopez, D. Angelov, Eur. Phys. J. Spec. Top. 147 (2007) 173.

[37] B. Alexandrov, Kim Ø. Rasmussen, A.R. Bishop, J. Biol. Phys. 35 (2009) 31.

[38] C.B. Tabi, A. Mohamadou, T.C. Kofane, J. Phys.: Condens. Matter 21 (2009) 335101. 